\title{
Endoscopic endonasal transsphenoidal direct access and Onyx embolization of a dural arteriovenous fistula mimicking a carotid-cavernous fistula: case report
}

\author{
Patrick J. Karas, MD, ${ }^{1}$ Robert Y. North, MD, PhD, ${ }^{1}$ Visish M. Srinivasan, MD, ${ }^{1}$ \\ Nathan R. Lindquist, MD, ${ }^{2}$ K. Kelly Gallagher, MD, ${ }^{2}$ Jan-Karl Burkhardt, MD, \\ Daniel Yoshor, MD, ${ }^{1}$ and Peter Kan, MD'1
}

${ }^{1}$ Department of Neurosurgery and ${ }^{2}$ Bobby R. Alford Department of Otolaryngology, Baylor College of Medicine, Houston, Texas

\begin{abstract}
The classic presentation of a carotid-cavernous fistula (CCF) is unilateral painful proptosis, chemosis, and vision loss. Just as the goal of treatment for a dural arteriovenous fistula (dAVF) is obliteration of the entire fistulous connection and the proximal draining vein, the modern treatment of CCF is endovascular occlusion of the cavernous sinus via a transvenous or transarterial route. Here, the authors present the case of a woman with a paracavernous dAVF mimicking the clinical and radiographic presentation of a CCF. Without any endovascular route available to access the fistulous connection and venous drainage, the authors devised a novel direct hybrid approach by performing an endoscopic endonasal transsphenoidal direct puncture and Onyx embolization of the fistula.
\end{abstract}

https://thejns.org/doi/abs/10.3171/2020.7.JNS201737

KEYWORDS dural arteriovenous fistula; carotid-cavernous malformation mimic; endoscopic endonasal transsphenoidal; percutaneous embolization; vascular disorders

$\mathrm{C}$ AROTID-CAVERNOus fistulas (CCFs) are shunts that allow blood to flow from the carotid artery (or branches thereof) into the cavernous sinus. ${ }^{1}$ The resulting arterialized cavernous sinus causes increased pressure in the superior ophthalmic vein, leading to venous congestion in the orbit. The classic clinical manifestation is unilateral chemosis, proptosis, and vision loss. However, dural arteriovenous fistulas (dAVFs) not involving the cavernous sinus can also manifest with similar clinical presentations if the venous drainage from the fistula causes increased pressure within the superior ophthalmic vein and subsequent vascular congestion within the orbit. ${ }^{2}$

Historically, CCFs were treated with either sacrifice of the offending carotid artery or surgical packing of the cavernous sinus. ${ }^{3}$ The modern treatment focuses on occlusion of the cavernous sinus through endovascular approaches, usually through a transvenous route. ${ }^{4,5}$ Similarly, the treatment for dAVFs is obliteration of the fistulous connection point and proximal venous drainage, either surgically or through an endovascular approach.

Here, we present the case of a woman with a dAVF whose clinical and radiographic presentation mimicked a CCF. Initial endovascular treatment failed due to a lack of venous access to the fistula and the inability to achieve obliteration of the fistula from a transarterial treatment.
We devised a hybrid approach utilizing an endoscopic endonasal transsphenoidal approach, allowing us to directly puncture the venous pouch under stereotaxy and endoscopic visualization, and completely embolize the fistula. To our knowledge, this is the first successful endoscopic endonasal transsphenoidal direct puncture and embolization of a dAVF.

\section{Case Report \\ History and Examination}

A 71-year-old right-handed woman was referred by an ophthalmologist to the emergency department with a 2-day history of acute-onset right eye pain, proptosis, and blurry vision. The patient's medical history was notable for warfarin use after cardiac valve replacement 7 years prior, aspirin use $(81 \mathrm{mg})$, chronic kidney disease now with normal estimated glomerular filtration rate, and a prior right-sided stroke with residual left upper-extremity spasticity. Her neurological examination was remarkable for right eye proptosis with conjunctival injection and an afferent pupillary defect with decreased visual acuity. The remainder of her cranial nerve examination, including extraocular movements, was unremarkable. No bruit could be auscultated from the orbit or temporal region.

ABBREVIATIONS CCF = carotid-cavernous fistula; dAVF = dural arteriovenous fistula.

SUBMITTED May 10, 2020. ACCEPTED July 1, 2020.

INCLUDE WHEN CITING Published online November 13, 2020; DOI: 10.3171/2020.7.JNS201737. 
CT angiography of the orbit and brain (Fig. 1) revealed dilated vascular channels in the region of the right cavernous sinus, anterior and medial to the cavernous internal carotid artery. The superior ophthalmic vein was also engorged and hyperdense. Cerebral angiography of the right common carotid artery showed no abnormalities. Left carotid injections (Fig. 2) showed venous filling during the arterial phase in the region of the right cavernous sinus with small indirect feeders from both internal and external carotid origins. The fistula was low flow and had no clear venous drainage through the superior or inferior petrosal sinus. Rather, contrast was seen to slowly diffuse into the proximal superior ophthalmic vein and proximal basilar plexus before fully dissipating. The distal superior ophthalmic vein appeared thrombosed.

Transvenous access via the right side was attempted; however, the right internal jugular vein was chronically occluded. The right cavernous sinus was then catheterized through the left internal jugular vein and left inferior petrosal sinus. Microcatheter injection of the cavernous sinus revealed a symmetric cavernous sinus and no connection to the venous fistula pouch (Fig. 2D and E). We therefore diagnosed the patient with a paracavernous, paraclinoid dural arteriovenous fistula, although a Barrow type D CCF with a loculated fistula pouch was also a possibility.

\section{Treatment}

Selective microcatheterization of the left internal maxillary artery was performed, gaining access just proximal to the arterial feeders to the fistula. We then performed Onyx (Medtronic) embolization of the fistulous branches with the aid of a dual-lumen Scepter C balloon (MicroVention) under inflation with the goal of pushing Onyx into the fistulous pouch. However, we were unable to reach the fistulous pouch with Onyx, resulting in incomplete treatment of the arteriovenous fistula. Postembolization angiography revealed persistent filling of the fistula primarily from small branches arising off the internal carotid artery.

After failure of the initial endovascular treatment, we devised a combined open and endovascular approach, with the goal of completely embolizing the fistulous pouch (Video 1).

VIDEO 1. Patient presentation, diagnostic imaging findings, and intraoperative video. Copyright Patrick Karas. Published with permission. Click here to view.

Informed consent was obtained, and the patient agreed to proceed with surgery. Surgery was performed in a hybrid operating room equipped with monoplane fluoroscopy. The patient's head was secured in a radiolucent skull clamp, and StealthStation (Medtronic) frameless stereotaxy was registered using the head CT angiogram. We performed an endoscopic endonasal transsphenoidal approach and removed the bone at the posterior sphenoid sinus overlying the venous pouch of the dAVF (Fig. 3). The exact location of the fistula was located using frameless stereotactic navigation based on the preoperative CT angiogram. Precise puncture of the fistula through the dura was important to avoid the adjacent cavernous segment of the internal carotid artery. We punctured the fistula
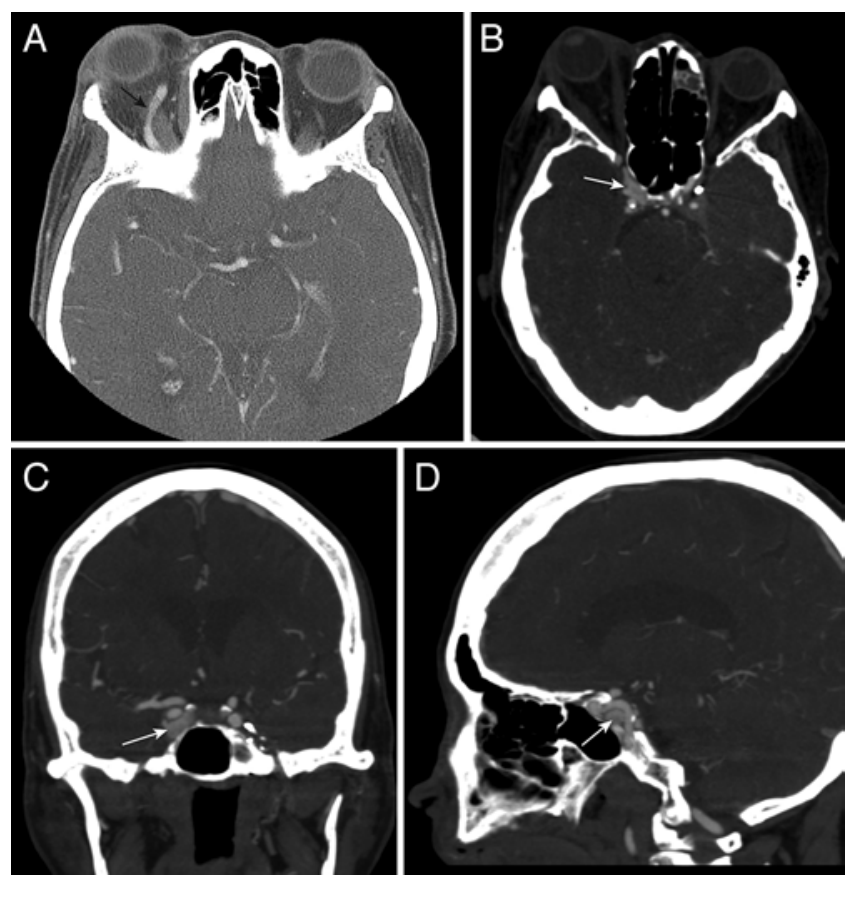

FIG. 1. Preoperative CT angiogram of the orbit (A) showing dilated hyperdense superior ophthalmic vein (black arrow). We hypothesize that this vein was the primary venous drainage of the fistula and that spontaneous occlusion of the vein led to the patient's acute onset of symptoms. Axial (B), coronal (C), and sagittal (D) brain CT angiograms showing the location of the fistula (white arrow) in the region of the right cavernous sinus just anterior and medial to the cavernous segment of the carotid artery. Copyright Patrick Karas. Published with permission.

with a 22-gauge spinal needle. Return of arterial blood as well as an intraoperative injection of contrast dye into the venous pouch confirmed proper location of the spinal needle within the fistulous pouch. We then proceeded to inject Onyx under live fluoroscopy until the fistula was completely obliterated. An intraoperative transradial angiogram of the left common carotid artery confirmed that we had completely obliterated the fistula.

\section{Postoperative Course}

By postoperative day 1, the patient noted significant improvement in her right eye pain, visual acuity, and proptosis. By postoperative day 5 , her proptosis and conjunctival injection was almost completely resolved (Fig. 4). The patient had an uneventful postoperative period. She was restarted on warfarin and a therapeutic heparin bridge on postoperative day 2 and discharged home on postoperative day 7 once her warfarin reached a therapeutic level. Her 3 -month postoperative visit showed complete resolution of her symptoms with no recurrence.

\section{Discussion}

Noncavernous dAVFs that mimic the clinical presentation of CCFs are rare, occurring in only $2 \%-15 \%$ of patients with arteriovenous fistulas and clinical examination findings suggestive of $\mathrm{CCF}^{2,6}$ As in our case, distinguishing a paracavernous dAVF from a true $\mathrm{CCF}$ can 
be difficult, even with direct microcatheterization of the cavernous sinus, and the diagnosis is presumptive despite a normal and symmetrical cavernous sinus on the venogram with no connection to the fistula. An isolated segment of the cavernous sinus that formed the venous pouch was in the differential diagnosis. In CCF mimics, although the fistulous connection is not at the cavernous sinus, the pathophysiology underlying the clinical symptoms of chemosis, proptosis, and vision loss is the same: downstream congestion, retrograde filling, and/or arterialized highflow blood in the superior ophthalmic vein causes venous congestion in the orbit and the resulting classic clinical phenotype?

In general, direct high-flow CCFs caused by traumatic or aneurysmal rupture classically present acutely, while indirect lower-flow CCFs present more gradually as the venous drainage pattern changes and pressure in the superior ophthalmic vein increases. ${ }^{5}$ Our patient presented acutely despite having a dAVF analogous to a Barrow type $\mathrm{D}$ indirect CCF. We hypothesize that the dAVF had been present but asymptomatic for a long period of time. Acute thrombosis of the superior ophthalmic vein (noted on the preoperative CT scan of the orbit) likely triggered an abrupt increase in intraorbital pressure from venous congestion, causing the patient's acute symptom onset.

The treatment of both CCF and dAVF focuses on identifying and obliterating the venous pouch of the fistula and proximal draining veins. ${ }^{8,9}$ Unfortunately, we had no transvenous access to the venous pouch to allow for embolization of it and the proximal draining vein. Transarterial access also failed as the small feeders arising off the distal internal maxillary were too narrow and long to reach the venous pouch with Onyx, and the feeders from the internal carotid artery were too small to safely embolize without a high risk of reflux into the internal carotid artery. Sacrificing the internal carotid artery is not an effective option, with the additional risk of hemiplegia and aphasia. We therefore considered other approaches.

Direct puncture of the superior ophthalmic vein can be used to access the cavernous sinus and treat CCFs. ${ }^{10,11}$ Thrombosis of the distal superior ophthalmic vein prevented attempting this approach in our case. Percutaneous embolization via direct puncture of the cavernous sinus through the superior orbital fissure has also been described. ${ }^{12,13}$ We decided against this approach because the venous pouch of the fistula was small and did not communicate with the cavernous sinus. While the superior orbital fissure provides access to the cavernous sinus, access to adjacent structures is less guaranteed; we were concerned that finding a small fistulous pouch adjacent to the cavernous sinus would be prohibitively difficult. Craniotomy was also considered but is significantly more invasive than our ultimate approach.

Given the proximity of the venous pouch to the posterior wall of the sphenoid sinus, we determined that a transsphenoidal approach would enable us to directly access the venous pouch for Onyx embolization. Direct puncture and embolization of the venous pouch has been successfully described for dAVFs of the skull base such as the hypoglossal canal, ${ }^{14}$ but not via an endoscopic endonasal transsphenoidal approach. Our ability to perform this ap-
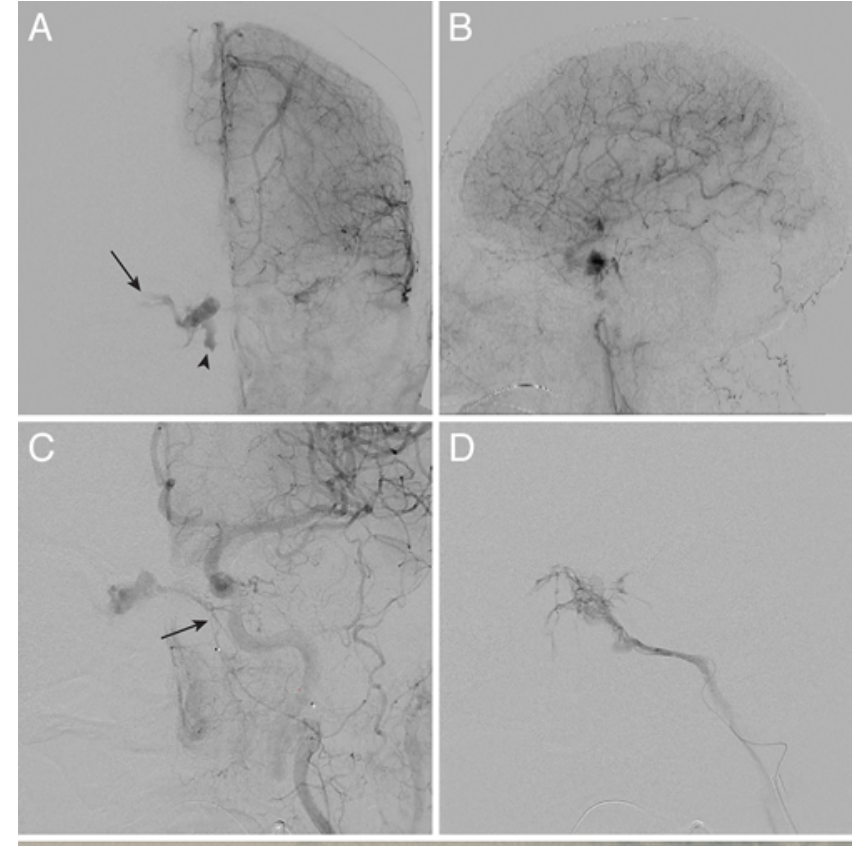

E

FIG. 2. A and B: Anteroposterior (A) and lateral (B) preoperative cerebral angiograms of the brain after left common carotid injection showing the fistula in the region of the right cavernous sinus. Note the origin of the right superior ophthalmic vein (black arrow) and the proximal basilar plexus (black arrowhead); there was no visualized distal contrast flow through these structures. C: Magnified left common carotid injection showing multiple very small feeding arteries arising directly from both the internal carotid artery and the internal maxillary artery (black arrow). D: Transvenous angiogram of the cavernous sinus via the left inferior petrosal sinus, revealing no connection to the fistula and a symmetric cavernous sinus, preventing endovascular access through a transvenous approach. E: Superimposed arterial and venous runs illustrate the juxtaposition of the arteriovenous fistula adjacent to the right cavernous sinus. Copyright Patrick Karas. Published with permission. Figure is available in color online only. 

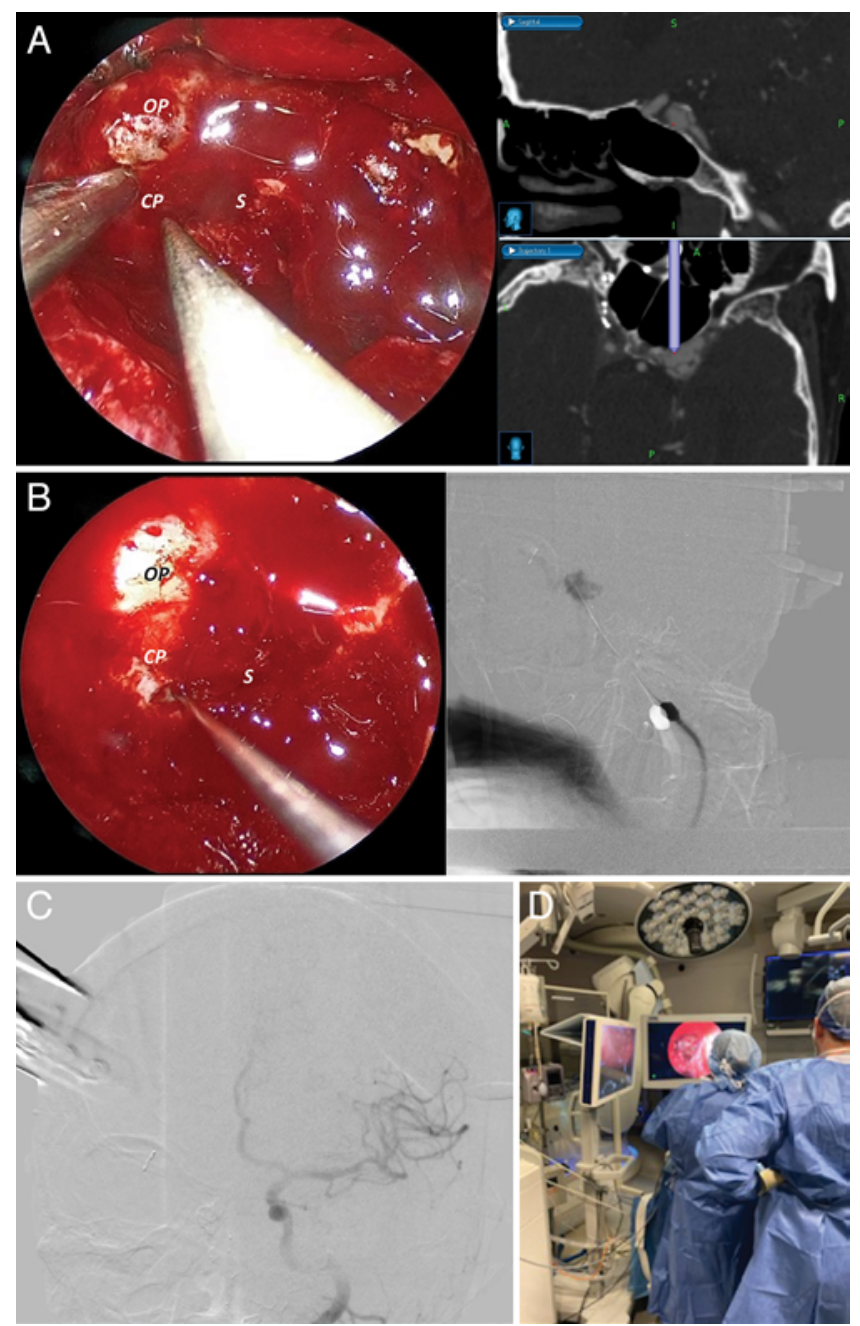

FIG. 3. A: Intraoperative frameless stereotactic navigation was used to localize the exact location of the arteriovenous fistula prior to bony removal. B: After the overlying bone was removed, a spinal needle was used to puncture the fistula under direct endoscopic visualization (left), contrast was injected to confirm entrance into the fistula pouch, and Onyx was injected through the spinal needle to embolize the fistula (right). C: An intraoperative postembolization angiogram confirmed that the fistula was completely obliterated. D: The endoscopic and frameless stereotactic monitors positioned above the patient's head had to be moved for the angiographic portion of the procedure in the hybrid operating room. $\mathrm{CP}=$ carotid protuberance; $\mathrm{OP}=$ optic protuberance; $\mathrm{S}=$ sella. Copyright Patrick Karas. Published with permission. Figure is available in color online only.

proach was dependent on a full understanding of the venous outflow of the fistula and the availability of a hybrid operating room.

Several technical challenges arose in the operating room. Because of the fixed operating room table, the patient was positioned supine in a reverse Trendelenburg position rather than the traditional beach chair position commonly used for transsphenoidal approaches. The StealthStation navigation terminal and endoscope monitor terminals were placed at the head of the bed. However, after puncturing the dAVF venous pouch, both the StealthStation and endoscopic monitor terminals had to
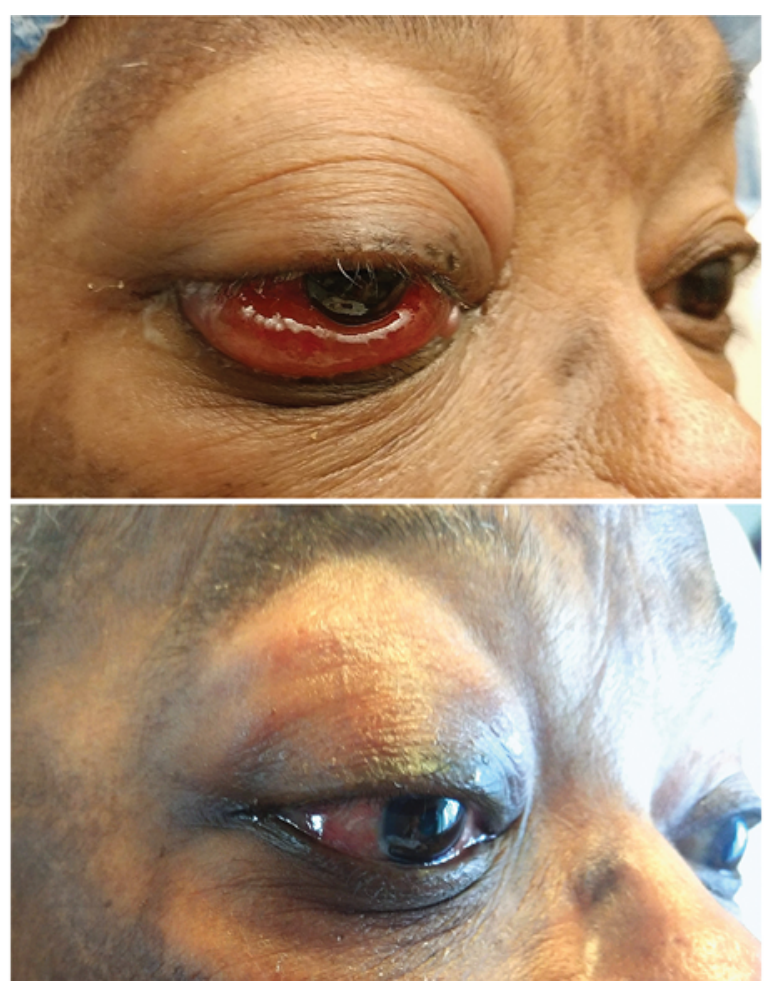

FIG. 4. Preoperative photograph (upper) showing ptosis, chemosis, and conjunctival injection. The patient's ocular symptoms resolved rapidly after surgery, with almost complete resolution by postoperative day 7 (lower), and complete resolution at her 3-month follow-up visit. Copyright Patrick Karas. Published with permission. Figure is available in color online only.

be moved to perform fluoroscopy. Stereotactic navigation was essential for localizing the venous pouch, which was adjacent to the cavernous sinus and carotid artery. Navigation enabled us to remove only the bone directly overlying the pouch to safely puncture the pouch rather than surrounding structures. We punctured the venous pouch with the spinal needle under direct endoscopic visualization while simultaneously confirming the location of the pouch with the navigation probe. After puncturing the fistulous pouch, the needle was held in place by hand while the StealthStation terminal and endoscope terminals were removed to bring in the monoplane. At this point, proper placement was confirmed by checking for continuous backflow of arterialized blood from the needle and subsequent injection of contrast into the venous pouch under fluoroscopy.

A second technical consideration was the proximity of the fluoroscope's cassette holder to the patient's nose. The cassette was too close to the nose to be able to attach the syringe directly onto the spinal needle. We therefore used a short segment of an intravenous extension tube, which is typically included in a lumbar puncture set. However, the volume needed to prime the intravenous extension tube with dimethyl suboxide (DMSO) is much greater than the volume generally needed during endovascular procedures, forcing us to prime the tube with two 1.0-ml volumes of DMSO prior to injecting Onyx. Similarly, more Onyx was 
needed to fill the dead space of the tubing. Standard intravenous tubing is $4 \mathrm{~mm}$ ( 0.16 inches) in outer diameter. The injection also needed to be performed expediently as DMSO was corrosive to the intravenous tubing.

Ultimately, the hybrid endoscopic endonasal transsphenoidal direct puncture and embolization of the dAVF was successful. The patient recovered rapidly from the procedure, and her ocular symptoms promptly resolved.

\section{Conclusions}

Paracavernous dAVFs can cause symptoms mimicking CCFs when the drainage pattern involves the cavernous sinus or the superior ophthalmic vein. Treatment of these lesions can be challenging if transvenous and transarterial routes are not favorable. We present a novel hybrid open and endovascular approach via endoscopic endonasal transsphenoidal direct puncture of the fistula to achieve complete embolization of the venous pouch and proximal venous drainage. Combining techniques and principles from endonasal and endovascular surgery allowed us to safely achieve a successful outcome with minimal morbidity to the patient.

\section{References}

1. Barrow DL, Spector RH, Braun IF, et al. Classification and treatment of spontaneous carotid-cavernous sinus fistulas. $J$ Neurosurg. 1985;62(2):248-256.

2. Kobkitsuksakul C, Jiarakongmun P, Chanthanaphak E, Pongpech S. Noncavernous arteriovenous shunts mimicking carotid cavernous fistulae. Diagn Interv Radiol. 2016;22(6): $555-559$.

3. Dorrance GM, Loudenslager PE. Physiological considerations in the treatment of pulsating exophthalmos. Am J Ophthalmol. 1934;17(12):1099-1111.

4. Chi CT, Nguyen D, Duc VT, et al. Direct traumatic carotid cavernous fistula: angiographic classification and treatment strategies. Study of 172 cases. Interv Neuroradiol. 2014; 20(4):461-475.

5. Ellis JA, Goldstein H, Connolly ES Jr, Meyers PM. Carotidcavernous fistulas. Neurosurg Focus. 2012;32(5):E9.

6. Hasuo K, Matsumoto S, Mihara F, et al. Dural non-cavernous sinus arteriovenous fistulas symptomatically simulating spontaneous carotid-cavernous fistulas: an analysis of angiographic findings. Radiat Med. 1997;15(4):203-208.

7. Sedat J, Kominami S, Siriwimonmas S, et al. Extracavernous arteriovenous fistulae. Report of five cases. Interv Neuroradiol. 1999;5(3):235-243.

8. Meyers PM, Halbach VV, Dowd CF, et al. Dural carotid cavernous fistula: definitive endovascular management and longterm follow-up. Am J Ophthalmol. 2002;134(1):85-92.
9. Ng PP, Higashida RT, Cullen S, et al. Endovascular strategies for carotid cavernous and intracerebral dural arteriovenous fistulas. Neurosurg Focus. 2003;15(4):ECP1.

10. Miller NR, Monsein LH, Debrun GM, et al. Treatment of carotid-cavernous sinus fistulas using a superior ophthalmic vein approach. J Neurosurg. 1995;83(5):838-842 .

11. Srinivasan VM, Sen AN, Kan P. Trans-superior ophthalmic vein approach for treatment of carotid-cavernous fistula. Neurosurg Focus. 2019;46(Suppl_2):V4.

12. Teng MMH, Lirng JF, Chang T, et al. Embolization of carotid cavernous fistula by means of direct puncture through the superior orbital fissure. Radiology. 1995;194(3):705-711.

13. Narayanan S, Murchison AP, Wojno TH, Dion JE. Percutaneous trans-superior orbital fissure embolization of carotid-cavernous fistulas: technique and preliminary results. Ophthal Plast Reconstr Surg. 2009;25(4):309-313.

14. Diaz OM, Toledo MM, Roehm JOF, et al. Unique percutaneous direct puncture technique for occlusion of a hypoglossal canal dural arteriovenous fistula. J Neurointerv Surg. 2018; 10(12):1179-1182.

\section{Disclosures}

The authors report no conflict of interest concerning the materials or methods used in this study or the findings specified in this paper.

\section{Author Contributions}

Conception and design: Kan, Karas, North, Gallagher, Burkhardt, Yoshor. Acquisition of data: Kan, Karas, North, Lindquist, Gallagher, Yoshor. Drafting the article: Kan, Karas. Critically revising the article: all authors. Reviewed submitted version of manuscript: all authors. Approved the final version of the manuscript on behalf of all authors: Kan.

\section{Supplemental Information \\ Videos}

Video 1. https://vimeo.com/442459088.

\section{Correspondence}

Peter Kan: Baylor College of Medicine, Houston, TX. ptkan@ utmb.edu. 